\title{
Stipendien, Förderpreise und neue Early Career Research Grants der GTH: Bewerbungsdeadline 30. November 2020
}

\begin{abstract}
In langer Tradition schreibt die GTH als wissenschaftlich-medizinische Fachgesellschaft jährliche Stipendien und Förderpreise aus, die jungen Wissenschaftlerinnen und Wissenschaftlern besondere Forschungsprojekte ermöglichen sowie hervorragende Forschungsarbeiten würdigen. Auch in diesem Jahr freuen wir uns auf Ihre zahlreichen Bewerbungen!
\end{abstract}

\section{Hans-Egli-Forschungsstipendium (30.000 €, Spende der Firma Bayer Vital)}

Das Stipendium soll dem/der Wissenschaftler/in ermöglichen, sich für ein halbes Jahr (bzw. für eine halbe Stelle ein Jahr lang) intensiv der Forschung eines Projektes auf dem Gebiet der angeborenen Blutungserkrankungen zu widmen.

\section{Rudolf-Marx-Stipendium \\ (bis zu $25.000 €$ )}

Mit dem Rudolf-Marx-Stipendium wird für deutschsprachige Wissenschaftlerlnnen ein umrissenes Forschungsprojekt aus dem Gebiet der Hämostaseologie oder der vaskulären Biologie/Medizin gefördert. Das Projekt muss an einer externen Forschungseinrichtung im Inoder Ausland durchgeführt werden. Das Stipendium ist als Anschub- oder Überbrückungsfinanzierung gedacht.

\section{Alexander-Schmidt-Preis (15.000€)}

Der Alexander-Schmidt-Preis wird für hervorragende Arbeiten auf dem Gebiet der Hämostaseologie vergeben. Für den Preis kann eine hochrangige Arbeit eingereicht werden, die von einem Gutachtergremium für die Veröffentlichung in einer wissenschaftlichen Zeitschrift angenommen oder bereits veröffentlicht worden ist. Die Arbeit soll aus dem Gebiet der Hämostaseologie oder vaskulären Biologie/Medizin kommen und ihre Veröffentlichung darf nicht länger als zwei Jahre vor dem Bewerbungszeitpunkt erfolgt sein.
Förderpreise für Nachwuchs-WissenschaftlerInnen:

Nachwuchsförderpreis

Thromboseforschung/vaskuläre

Medizin (2.500€)

auf dem Gebiet der Hämostaseologie, Schwerpunkt Thromboseforschung.

\section{Nachwuchsförderpreis}

Blutungskrankheiten (2.500€)

auf dem Gebiet der Hämostaseologie, Schwerpunkt Blutungskrankheiten.

Es können wissenschaftliche Arbeiten eingereicht werden, die von einem Gutachtergremium für die Veröffentlichung in einer wissenschaftlichen Zeitschrift angenommen oder bereits veröffentlicht worden sind. Die Arbeit soll aus dem Gebiet der Thromboseforschung/ vaskulären Medizin oder aus dem Gebiet Blutungskrankheiten kommen und ihre Veröffentlichung darf nicht länger als zwei Jahre vor dem Bewerbungszeitpunkt erfolgt sein.

Neu:

Early Career Research Grants of the Society of Thrombosis and Haemostasis (3 x $100.000 €$ p.A.) Ab 2021 vergibt die GTH jährlich, befristet auf drei Jahre, bis zu drei Early Career Research Grants in Höhe von bis zu 100.000,00 $€$ an NachwuchsforscherInnen.

Voraussetzung für Bewerbungen ist ein inhaltlicher Fokus in den Bereichen Hämostase, Thrombose oder vaskuläre Biologie mit einem experimentellen, translationalen oder klinischen Forschungsansatz. Der Arbeitsmittelpunkt sollte in den GTH Ländern liegen (Deutschland, Österreich, Schweiz), gern gesehen werden "collaborative» Projekte, z.B. Kooperationen zwischen GTH Ländern oder Kooperationsprojekte zwischen Forschergruppen.

\section{Antragstellung}

Für alle Stipendien und Preise sind bei der Antragstellung individuelle Formalien sorgfältig zu beachten. Detaillierte Informationen, Merkblätter und Formulare finden sie auf der Webseite www.gth-online. org. unter der Rubrik "Förderung".

\section{Infos zur Bewerbung}

Antragsformulare sowie detaillierte Informationen zur Ausschreibung der Preise stehen auf der Webseite der GTH für Sie bereit. Stichtag für die Einreichung aller Bewerbungen ist der 30. November. Bis zu diesem Termin müssen Ihre Unterlagen in digitaler Form per Mail unter Angabe aller erforderlichen Daten in der Geschäftsstelle der GTH vorliegen. Gerne können Sie Ihre Bewerbung auch bereits früher einsenden. Bei Fragen zum Bewerbungsprozess und zu den Unterlagen unterstützen wir Sie gerne und stehen Ihnen telefonisch und per Mail zur Verfügung.

GTH Geschäftsstelle

0221-42334626

mail@gth-online.org

www.gth-online.org

\section{Preisverleihung}

Normalerweise findet die Preisverleihung jeweils im Februar bei der Eröffnungsveranstaltung des GTH Jahreskongresses statt, die Entscheidung über die Vergabe trifft der Vorstand am Vorabend des Kongresses. Als Bewerber ist die Teilnahme an der Eröffnungsveranstaltung des Jahreskongresses verpflichtend.

2021 ist jedoch alles anders, denn der GTH Jahreskongress wird als Online-Tagung stattfinden. Die Planung zur Preisvergabe ist noch nicht abgeschlossen und wir halten Sie auf der Webseite der GTH, in unseren Newslettern sowie in den kommenden Ausgaben der Zeitschrift auf dem Laufenden! 


\section{Bienvenue...}

...zur 65. Jahrestagung der Gesellschaft für Thrombose- und Hämostaseforschung. Erstmals online!

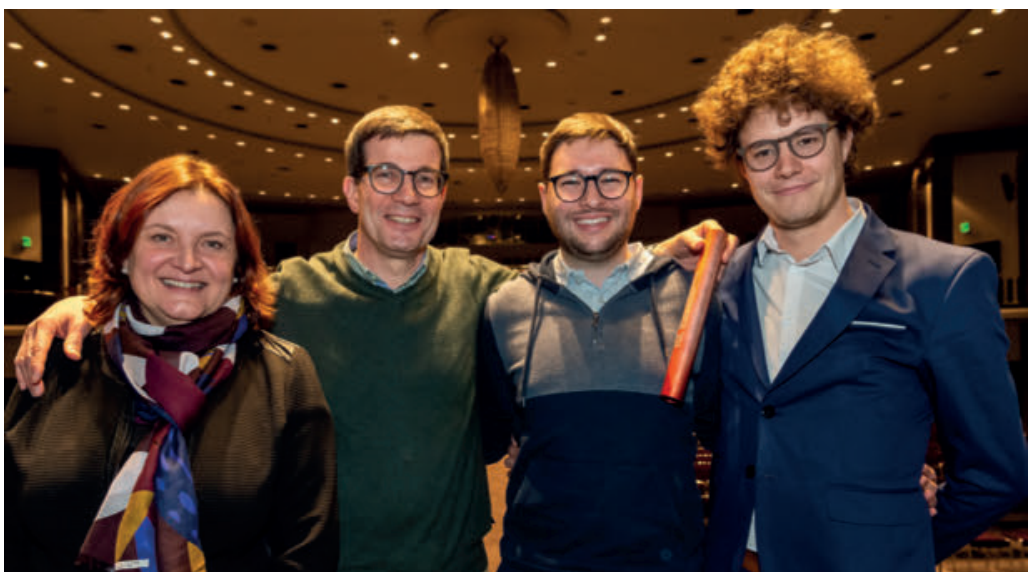

Das lokale Organisationskomitee:

Dr. phil. nat. Debora Bertaggia Calderara, Prof. Dr. med. Lorenzo Alberio,

MSc. Ing. Alessandro Aliotta PhD-Sudent, Dr. med. Maxime G. Zermatten.

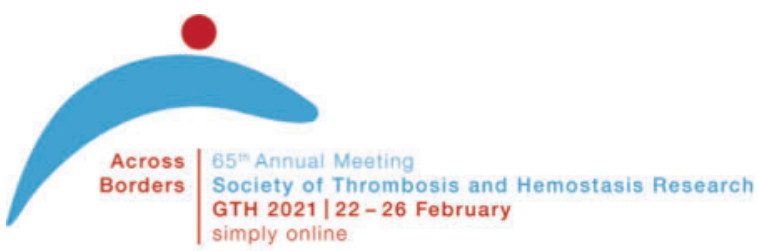

www.gth2021.org

\section{ACROSS (NEW) BORDERS}

Zum ersten $\mathrm{Mal}$ in seiner Geschichte sollte der GTH Kongress in der Westschweiz stattfinden: „La Romandie“.

Ich hatte wirklich gehofft, dass dieser attraktive Tagungsort es den Teilnehmerinnen und Teilnehmern aus der Schweiz, aus Deutschland und Österreich, Frankreich und Italien und möglicherweise auch aus anderen europäischen Ländern ermöglicht hätte, sich in Lausanne für vier Tage der Wissenschaft und der klinischen Praxis auf dem Gebiet der Hämostase zu widmen.

Das klitzekleine SARS-CoV-2 änderte unsere großen Pläne. Wegen der andauernden COVID-19-Pandemie zogen wir ein Hybrid-Treffen in Betracht, aber wir mussten uns schließlich für einen ausschließlich online stattfindenden Kongress entscheiden. Auch wenn diese Entscheidung einige schmerzliche Verzichte mit sich bringt, wird sie ebenso neue Möglichkeiten eröffnen.

Wir werden die wunderbare Umgebung von Lausanne mit ihren Weinbergen, den Bergen und dem See vermissen. Wir werden die Begegnungen und lebhaften Diskussionen vermissen. Wir werden Freunde und Kollegen vermissen.
Andererseits werden wir vom digitalen Netzwerk profitieren. Bestenfalls wird der Online-Kongress Teilnehmer anziehen, die an einem „physischen“ GTH Kongress nicht teilgenommen hätten und so unser Publikum erweitern. Für Abstracts und Oral Communications werden Online-Diskussionsforen eröffnet, welche die Interaktion mit den Autoren während des gesamten Kongresses ermöglichen werden. Die lebendige 3D-Industrieausstellung wird einen ganzen Monat lang geöffnet sein. Das Online-Kongressformat wird das Problem der parallelen Sitzungen lösen: Alle Präsentationen werden online verfügbar sein, sodass Sie diese nach Belieben anhören können. Sie könnten sogar einen „Bier- \& Pizza-Workshop“ organisieren und dabei Ihre bevorzugten Sessions mit Ihrem Team genießen ... Denken Sie einfach ACROSS BORDERS und lassen Sie uns das Beste aus der Situation machen!

\section{ACROSS (OLD) BORDERS}

Die Hämostaseologie ist einfach faszinierend! Meiner Erfahrung nach liegt einer der Gründe dafür in ihrem interdisziplinären Charakter, den wir täglich in der Diskussion klinischer Fälle mit Kollegen anderer medizinischer Fachrichtungen verwirklichen: in der Notaufnahme und im Operationssaal, auf der Kinderstation, im Labor und in so unterschiedlichen Bereichen wie der Hepatologie, der Geburtshilfe oder der Hämatologie.

Der GTH Kongress 2021 konzentriert sich auf interdisziplinäre Aspekte der Hämostaseologie, öffnet die Türen für Kollegen aus anderen medizinischen Fachgebieten und öffnet den Blick für andere Sichtweisen und Denkweisen der Hämostaseologie.

\section{ACROSS BORDERS}

Wir haben einen privilegierten Beruf! Täglich begegnen wir Menschen in recht verletzlichen Phasen ihres Lebens - das ist unsere größte Verantwortung. Wenn wir forschen, können wir Wissen schaffen- das ist unsere größte Herausforderung.

Klinische Leidenschaft und Forschung! Mein erster medizinischer Lehrer, Carlofelice Beretta-Piccoli (1946-1994), pflegte uns jungen Assistenzärztinnen und Assistenzärzten zu sagen und durch sein eigenes Berufsleben zu veranschaulichen, dass ein versierter Arzt intellektuell neugierig, wissenschaftlich genau und menschlich in der Lage ist, „(di) appassionarsi al destino del paziente". 
Das Motto der 65. Jahrestagung der Gesellschaft für Thrombose- und Hämostaseforschung - ACROSS BORDERS - steht für all dies: Internationalität, interdisziplinäre Hämostase, Wissenschaft und Humanität ...und die digitale Barriere.
Wir - das lokale, schweizerische und internationale Organisationskomitee - haben uns dafür eingesetzt, die Rahmenbedingungen für einen erfolgreichen wissenschaftlichen Kongress zu schaffen. Aber nur, wenn Sie Ihre besten
Abstracts einreichen und sich aktiv beteiligen, wird der Kongress „, bereichernd und denkwürdig" ...auch wenn er nur online stattfindet

\section{Bienvenue!}

\section{Ihr Lorenzo Alberio}

Médecin chef

Centre Hospitalier Universitaire Vaudois

Service et Laboratoire central d'hématologie

Rue du Bugnon 46

$\mathrm{CH}-1011$ Lausanne

lorenzo.alberio@chuv.ch

\section{Kongressinformationen}

\section{Ablauf}

Mo. 22.02.2021

18.00 - 20.00 Uhr Opening Ceremony

Di. 23.02. - Fr. 26.02.2021

12.00 - 13.30 Uhr Satellite Symposia

13.45 - 15.15 Uhr Oral Communications

15.30 - $16.30 \mathrm{Uhr}$ Plenary

16.45 - 18.15 Uhr State of the Art

18.30 - 20.00 Uhr Satellite Symposia

\section{Abstract Topics}

\section{Subject area}

- Acquired bleeding disorders

- Antithrombotic treatment

- Arterial thromboembolism

- Coagulation and fibrinolysis

- COVID-19

- Crosstalks between hemostasis and other systems

- Diagnostics and laboratory tests

- Hereditary bleeding disorders

- In vitro and in vivo models of hemostasis

- Innovation and Novelties

- Platelets- Disorders of platelet function and numbers

- Platelets- Physiology

- Vascular wall biology and disorders

- Venous thromboembolism

- von Willebrand factor and ADAMTS13

- Miscellaneous
Anmeldung

Online seit 1. Juli 2020

Abstract Submission

Online seit 1. Juli 2020

geöffnet bis Sonntag, 11. Oktober 2020 (12.00 Uhr)

Info und Kontaktt

www.gth2021.org

\section{Interdisciplinary area}

- Anesthesiology

- Angiology

- Basic research

- Cardiology

- Clinical practice

- Clinical research

- Gynecology/Obstetrics

- Hemato-oncology

- Hepatology/Gastroenterology

- Intensive care

- Laboratory medicine

- Laboratory technology

- Neurology

- Pediatrics

- Transfusion medicine

- Trauma/Surgery 


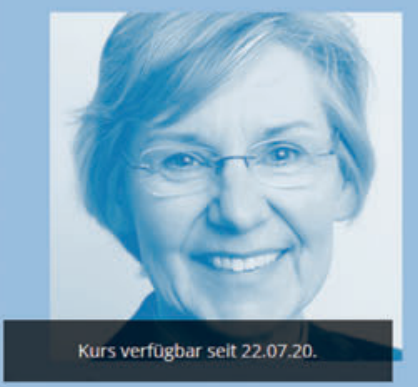

\section{GTH Mediathek -}

\section{Das Wissensportal der Hämostaseologie}

\section{Nach dem Start der GTH Akademie Mediathek Ende Juni sind inzwi- schen einige hundert registrierte Teilnehmerlnnen auf der digitalen Plattform angemeldet und informieren sich über aktuellste Erkenntnis- se aus der Hämostaseologie.}

Die bisherigen Rückmeldungen zur GTH Mediathek bestätigen vor allem, dass das Format (ReferentIn und Präsentation zeitgleich im Video) und die kurzen Laufzeiten, pro Kurs 3 Lektionen mit ca. 10 bis 15 Minuten, den Teilnehmerlnnen viel Freiraum zur individuellen Gestaltung Ihre Weiterbildung lassen.

Die GTH Geschäftsstelle informiert alle Interessierten regelmäßig per Newsletter über neu eingestellte Beiträge und die Termine zu den Live Q\&A Sessions. Die ca. 1-stündigen, moderierten Live-Diskussionen mit den ReferentInnen finden 1-2 Wochen nach der Veröffentlichung der Beiträge jeweils mittwochs um $16.00 \mathrm{Uhr}$ statt und bieten die Möglichkeit, Fragen zu stellen und sich zu Inhalten zu äußern. Falls Sie noch

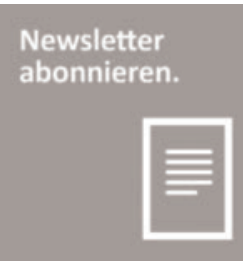

nicht im Verteiler sind, können auch Sie sich auf der Webseite gerne für unseren Newsletter registrieren. Klicken Sie auf den Button "Newsletter abonnieren“ und geben Sie Ihren Namen und die E-Mail-Adresse an.

Bei Interesse finden Sie im Menü „Alle Themen" auch das vollständige Programm mit allen noch geplanten Beiträgen.

Und wenn Sie sich ebenfalls registrieren möchten, fügen Sie das GTH Mediathek Ticket in den Warenkorb und schließen den Kauf ab. Es stehen mehrere Zahlungsarten zur Verfügung. Die Schutz-

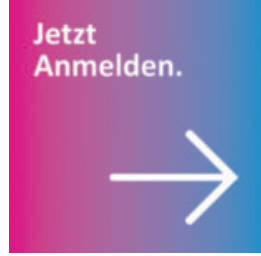

gebühr in Höhe von 25,00 € berechtigt Sie bis Mai 2021, alle Beiträge zu sehen. Nach kurzer Lernerfolgskontrolle erhalten Sie sofort Ihre Zertifikate. Wir übermitteln Ihre CME-Punkte an die zuständige Landesärztekammer.

Übrigens werden die 25,00 € bei einer Anmeldung zur Präsenzveranstaltung der GTH Highlights 2021 in voller Höhe angerechnet.

Weitere Infos auf der Webseite: www.gth-mediathek.org 


\section{Zur Verfügung stehen bereits folgende Kurse, zertifiziert mit jeweils 2 CME-Punkten:}

Hämostasestörungen

bei Covid-19

Prof. Dr. Florian Langer

Universitätsklinikum

Hamburg-Eppendorf,

Zentrum für Onkologie,

II. Med. Klinik und Poliklinik

\section{Erworbene Hämophilie}

Prof. Dr. Andreas Tiede

Medizinische Hochschule

Hannover,

Klinik für Hämatologie,

Hämostaseologie, Onkologie und

Stammzelltransplantation

\section{Antikoagulation}

bei Kindern

\section{Prof. Dr. Christoph Male}

Medizinische Universität Wien,

Univ.-Klinik für Kinder- und

Jugendheilkunde
Antiphospholipid-

Antikörpersyndrom

Prof. Dr. Ingrid Pabinger

Medizinische Universität Wien,

Klinische Abteilung für

Hämatologie und Hämostaseologie

\section{Heparin-induzierte}

Thrombozytopenie

Prof. Dr. Andreas Greinacher

Universitätsmedizin Greifswald,

Abteilung Transfusionsmedizin

Halbwertszeitverlängerte

Faktorenkonzentrate in der

Hämophilie A (spons. Bayer)

Prof. Dr. Bettina Kemkes-Matthes

Universitätsklinikum Giessen und

Marburg GmbH,

Interdisziplinärer Schwerpunkt

für Hämostaseologie
Von-Willebrand-Syndrom

PD Dr. Jan-Dirk Studt

Universitätsspital Zürich,

Klinik für Medizinische Onkologie

und Hämatologie

Hämophilie A:

Gelenkgesundheit heute und

Ausblick in die EHL Zukunft

(spons. SOBI)

PD Dr. Robert Klamroth

Vivantes Klinikum im Friedrichshain, Berlin,

Klinik für Innere Medizin,

Hämophiliezentrum

Hämophilie B

Dr. Carmen Escuriola-Ettingshausen Hämophilie Zentrum Rhein Main Mörfelden-Walldorf

\section{Diese Kurse sind in der Produktion und werden in Kürze, ebenfalls mit 2 CME-Punkten zertifiziert, in die Fortbildungsreihe aufgenommen:}

\section{Emicizumab bei Kindern \\ mit Hämophilie A \\ (spons. Chugai/Roche) \\ Dr. Ivonne Wieland}

Medizinische Hochschule Hannover,

Pädiatrische Hämatologie und

Onkologie

\section{Antikoagulanzien}

PD Dr. Jan Beyer-Westendorf,

Universitätsklinikum Carl Gustav

Carus an der Technischen

Universität Dresden,

Medizinische Klink I,

Bereich Hämatologie und

Hämostaseologie

\section{Antithrombozytäre Substanzen}

\section{Prof. Dr. Daniel Dürschmied}

Universitäts-Herzzentrum Frei-

burg-Bad Krozingen, Kardiologie und Angilogie I und internistische Intensivmedizin

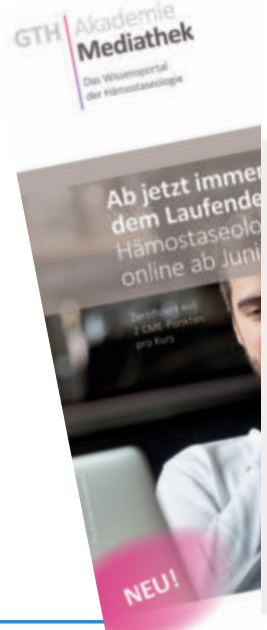
Mediathek onmunser.

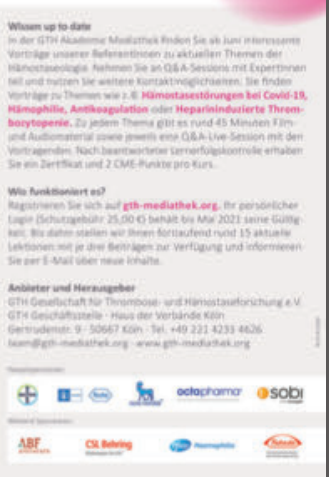




\section{Zertifizierung der Hämophilie-Zentren}

Am 1. September 2020 trat die Neuregelung der Arzneimittelversorgung von Hämophilie-Patienten in Kraft. Das Interesse der GTH an der Zertifizierung von Hämophilie-Zentren begründet sich unter anderem durch das neue GSAV - die zertifizierten Zentren werden in diesem Rahmen zukünftig eine besondere Bedeutung haben.

Nachdem die GTH innerhalb kurzer Zeit mit der Firma ClarCert einen qualifizierten Kooperationspartner verpflichtet hatte, wodurch zahlreiche GTH Fachexpertinnen und Fachexperten als AuditorInnen rekrutiert und geschult werden konnten, erfolgte Anfang 2020 die Zertifizierung des ersten Fachzentrums (Hämophilie-Zentrum Homburg-Saar). Im März gab es jedoch durch die Co-
vid-19-Pandemie einen jähen Stopp des erfolgreichen Laufes!

Zunächst mussten etliche bereits terminierte Audits bedauerlicherweise verschoben werden, inzwischen nimmt das Projekt wieder Fahrt auf. Unter sorgfältiger Beachtung aller Hygiene- und Abstandsregeln und selbstverständlich auch der Kapazitäten der Zentren werden in den nächsten Wochen weitere vier Einrichtungen auditiert.

Weiterhin steht durch ein Sponsoring für 2020 und voraussichtlich auch für 2021 ein zweckgebundenes Budget zur Verfügung, um die Vorabberatung interessierter Hämophilie-Zentren zu ermöglichen. Im Rahmen des Projektes wird den Zentren ein/e Berater/in zur Verfü- gung gestellt, um das Antragsformular und die Anmeldung zur Zertifizierung zu prüfen und zu begleiteten. Aktuell nehmen sieben Institute diese Beratung für die anschließende Zertifizierung in Anspruch.

Für Anfragen zur kostenlosen Vorabberatung wenden sie sich bitte an die GTH Geschäftsstelle:

mail@gth-online.org

Tel. 0221-42334626

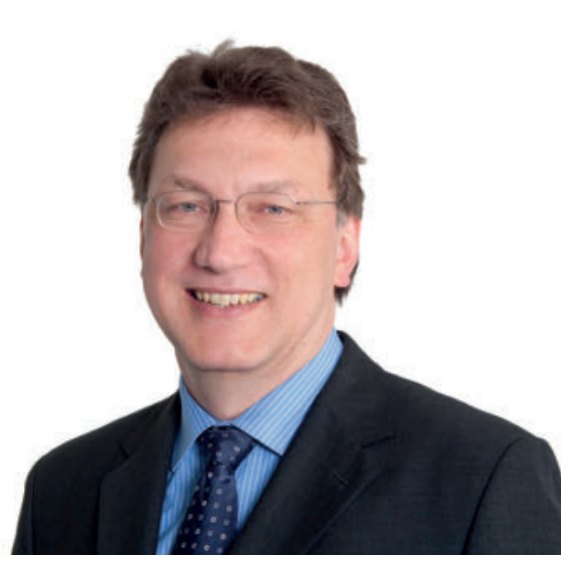

Delegierter Leitlinien- und Stellungnahmebeauftragter der GTH:

Prof. Dr. med. Wolfgang Korte CEO und Chefarzt

Hämostase- und Hämophilie-Zentrum Hämatologisches Ambulatorium St.Gallen Wolfgang.Korte@zlmsg.ch

\section{Leitlinien und Konsensus-Papiere:}

\section{Effizientes Engagement der GTH}

Die FachexpertInnen und Mitglieder der GTH arbeiten regelmäßig an Leitlinien und Stellungnahmen in ihren und angrenzenden medizinischen Fachbereichen. Nach einem Vorstandsbeschluss in diesem Jahr sind alle Beteiligten aufgefordert, ihre aktuellen und zukünftigen Arbeiten zu Leitlinien, Stellungnahmen und Empfehlungen in einem zentralen Prozess über die GTH Webseite mit unserem Leitlinienbeauftragten Prof. Dr. Wolfgang Korte abzustimmen.

Inzwischen wurden die bisher publizierten GTH Leitlinien und sonstigen Do- kumente auf der Webseite unter dem Menüpunkt „Fachexperten“ zentral veröffentlicht. Dort finden Sie nun auch ein Formular zum Download, mit dem Sie Ihre jeweiligen Projekte bitte bei Prof. Korte anmelden. Damit möchten wir die Möglichkeit einer effizienten und kollaborativen Erstellung und Veröffentlichung nutzen.

Bei Fragen wenden Sie sich gerne an die Geschäftsstelle: mail@gth-online.org

Tel. 0221-42334626

\section{Impressum}

Verantwortlich für den Inhalt der GTH News:

GTH

Gesellschaft für Thromboseund Hämostaseforschung e.V.
GTH Geschäftsstelle

Haus der Verbände

Gertrudenstr. 9

50667 Köln
Tel. 0221-42334626

mail@gth-online.org www.gth-online.org 